\title{
Adaptation and security validation of an irradiator suitable for use of cesium-137 sources
}

\author{
Rogério M.V. Silva, ${ }^{1 a}$ Walmir Belinato, ${ }^{1,2}$ William S. Santos, ${ }^{1}$ Danilo O. Junot, ${ }^{1}$ Luiza F. Souza, ${ }^{1}$ \\ Divanízia N. Souza ${ }^{1}$ \\ ${ }^{1}$ Department of Physics, Federal University of Sergipe - UFS, São Cristóvão, Sergipe, Brazil \\ ${ }^{2}$ Federal Institute of Bahia - IFBA, Vitória da Conquista, Bahia, Brazil
}

\begin{abstract}
With the aim of offering a new application for ${ }^{137} \mathrm{Cs}$ sources that were previously used in brachytherapy, we developed an irradiator for TL and OSL dosimeters by adapting a shielded storage container; this irradiator was named SOPHIA. It was assembled from a cubic iron casing, filled with lead, with external dimensions of $25 \times 25$ $\times 25 \mathrm{~cm}^{3}$. It has three different drawers with two sample holders suitable for samples or dosimeters with dimensions of up to $2 \mathrm{~cm}$. Dose distribution of the surface of sample holder was obtained with EBT-3 radiochromic film and detectors thermoluminescent CaSO4: Dy. Regarding the security conditions of irradiation, the whole routine procedure for placement and removal of samples was simulated using Monte Carlo code. The equipment presented proper security conditions and the doses received by users during procedures for placement and extraction of the samples are in accordance with international standards.
\end{abstract}

\section{Introduction}

The use of radioactive materials in the form of seeds, needles, and wires for treatment of cancer has been widespread since the early nineteenth century. Various types of radionuclides, such as radium226, cesium-137, cobalt-60, and iridium-192 were, and still are, used for this purpose [1]. The selection of a particular radionuclide for use in brachytherapy depends on several factors; however, flexibility, which allows the production of suitable irradiation devices as treatment requires, high specific activity, which enables the miniaturization of these objects, and the absence of photons with very high energies due to the conditions of radioprotection are the main characteristics of an ideal radionuclide [2]. Based on these requirements, cesium-137 needles have been replaced by ${ }^{192} \mathrm{Ir}$ sources, which are coupled to post-loading devices, allowing greater protection of staff and patients; as the gamma rays of ${ }^{192} \mathrm{Ir}$ have an average energy of $380 \mathrm{keV}$, which is below the energy of $662 \mathrm{keV}$ from photons of ${ }^{137} \mathrm{Cs}$. In this context, many hospitals have inoperable ${ }^{137} \mathrm{Cs}$ sources in their deposits with relatively high dose rates because the half-life of this radionuclide is approximately 30 years. In

\footnotetext{
${ }^{\text {a }}$ Corresponding author: rmv.fisica@gmail.com
} 
order to keep these sources under appropriate conditions of radioprotection, periodic supervision is required, which can be a burden for these institutions. Thus, this paper presents a set of techniques for the adaptation, assembly, and verification of safety conditions of a radiator suitable for ${ }^{137} \mathrm{Cs}$ needles, which can be used by research centers, such as universities, in assessing the behavior of materials in the presence of gamma radiation and for the development of new dosimeters.

\section{Materials and Methods}

To mount the radiator prototype, which was named SOPHIA, we used a shielded storage container formed by a 0.5 -cm-thick iron shell with cubic dimensions of $25 \times 25 \times 25 \mathrm{~cm}$ completely filled with lead. The container used has three drawers to accommodate the cesium needles. The drawers are 18 $\mathrm{cm}$ long and are formed by a movable iron base which supports a piece of lead to assist in shielding. To mount the irradiation set-up, six acrylic parts were made, $1 \mathrm{~cm}$ in height, $2 \mathrm{~cm}$ wide, and $10 \mathrm{~cm}$ long, with two orifices of $0.5 \mathrm{~cm}$ radius and $2 \mathrm{~mm}$ depth positioned at one end in which the samples were placed. In order to position the needles of cesium-137 in relation to the samples, three pieces of acrylic were prepared in the form of a T-shape with one, three, and eight holes at the upper end, respectively. Then, the sources were introduced into the holes, and the acrylic pieces were fixed at the bottom and ceiling of the placement location of the drawers inside the container. Figure 1 shows the layout of the radiator side section.

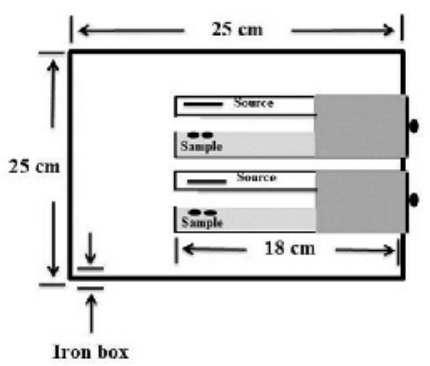

Figure 1. Design of the radiator in coronal section.

Radiochromic films have been widely used in dosimetric procedures, where it is necessary to know the planar behavior of doses [3][4]. Accordingly, to determine the distribution of doses on the surface of the sample holder, radiochromic films of the EBT-3 generation from ISP were used. Pieces of films, with dimensions similar to the placement position of the samples, were fixed on the acrylic base secured to the drawer. The films were then introduced into the container for irradiation three times with different exposures for each drawer.

The Monte Carlo method has been used in numerous situations that present risks associated with the handling and use of radioactive sources [5-7]. Due to their small size, low fading, and easy reading, nanoDot OSL dosimeters based on A12O3:C from Landauer have also been used in these types of situations [8][9]. Thus, in order to certify the safety conditions of irradiation, the routine handling procedure was simulated using the transport code MCNPX 2.7 and the DEXRAX32 spectrum generator [10][11]. The voxel computational simulator of ICRP-110 was used to represent the user with a dosimeter positioned in the region of the left chest and the front of the eye to estimate the dose to the whole body and lens, respectively [12]. The results were compared with doses obtained by nanoDot dosimeters positioned in the chest of a physical phantom [13]. The total exposure time was $3 \mathrm{~min}$, which was related to an average of 25 manipulations of placement and removal of samples. A Microstar reader (Landauer) was used to read the dosimeters. Pellets of CaSO4:Eu,Ag developed in our research center exhibit suitable behavior for thermoluminescent radiation dosimetry [14]. Accordingly, to verify the sample irradiation performance of the radiation, pellets of CaSO4:Eu,Ag were prepared, $1 \mathrm{~mm}$ thick and $5 \mathrm{~mm}$ in diameter. They were placed in the holes in the sample holder and irradiated for different times. 


\section{Results and Discussion}

The drawers are relatively light and easy to handle. Figure 2 shows the sampler tray and the irradiation set-up with the T-shaped base suitable for eight needles properly positioned.

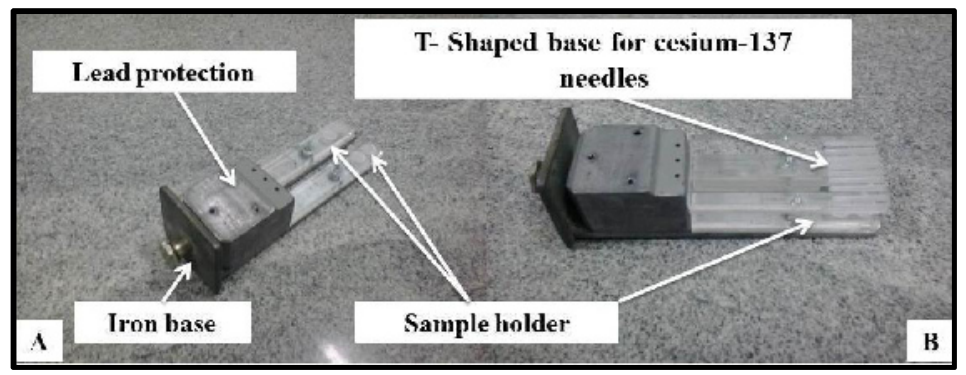

Figure 2. A) Drawer of the sample holder; B) sampler tray and T-shaped base suitable for eight needles.

The planar dose distributions obtained from the radiochromic films allowed us to know the dose deposition behavior on the sample holder surface. Figure 3 shows the dose distribution after $12 \mathrm{~h}$ of exposure in the right sample holder of the drawer that has a single needle. The area underlined represents the projection of the holes where the sources are positioned and the dose behavior versus exposure time for the right side of drawer 1, containing only one source.
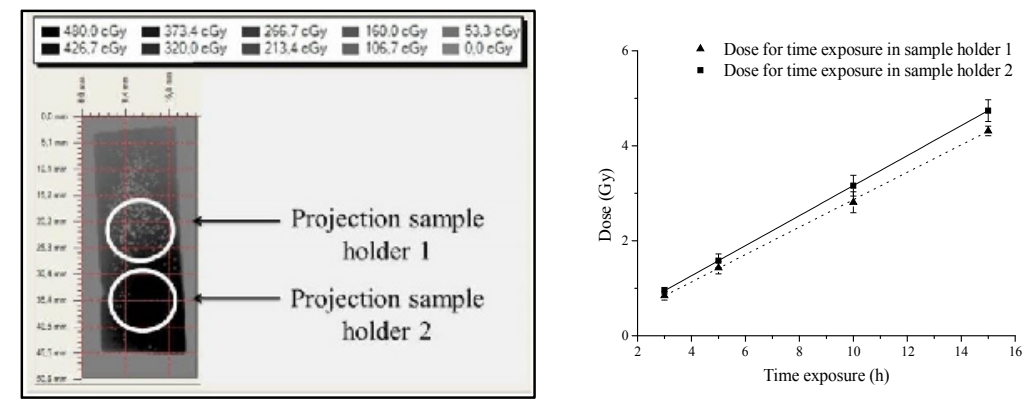

Figure 3. Dose distribution in the sample holder after $12 \mathrm{~h}$ of exposure and projection in the sample holder from the drawer that has one needle and dose behavior versus exposure time for sample holders 1 and 2, inside the drawer that contains only one source

From the results of the dose distributions we observed that there is a moderate dose inhomogeneity on the surface of sample holder 1.

A difference was identified between the dose deposition behaviors in sample holders 1 and 2. For example, the results of irradiation of a sample for 10 hours in sample holder 1 corresponded to a dose of about $2.8 \mathrm{~Gy}$, whereas with the same exposure time in sample holder 2 we obtained a dose of 3.1 Gy. This different behavior was linear for all exposure times studied, with the dose deposited on sample holder 1 being $11 \%$ lower, on average, that the dose on sample holder 2 . This characteristic can be explained by the high dose gradient of these types of sources and the different spatial arrangements of the holes in which the samples were placed with respect to the position of the source. It can also be seen that the difference between the absorbed doses may vary depending on the placement of the sample in the hole. However, materials with a circular dimension of $0.785 \mathrm{~cm}^{2}$, which corresponds to the total area of the hole, can offer better conditions of higher reproducibility. These characteristics were identified for the three drawers made.

Simulations of a user manipulating the irradiator, conducted by Monte Carlo code and a physical phantom, allowed the safety conditions of the irradiator to be verified. Figure 5 shows the scheme of the simulation performed. 


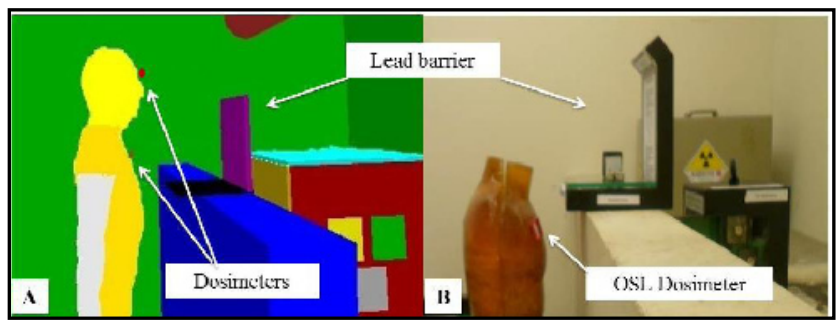

Figure 5. Simulation of procedures for handling the irradiator: A) computational phantom; B) physical phantom.

The results obtained by Monte Carlo simulation and by OSL dosimeters positioned in a physical phantom after an exposure time of three minutes, equivalent to approximately 25 manipulations, showed effective doses much lower than the limits established in the applicable regulations with respect to equivalent/effective dose limits for individuals from the public and occupationally exposed [15].

\section{Conclusions}

The adaptation techniques used to produce the irradiator prototype SOPHIA are feasible and can give functionality to the old needles of cesium-137, giving research centers access to monoenergetic photon beams of known dose rates. The manipulation procedure of the irradiator is simple, allowing the exposure time of the user to be reduced. Even when the techniques described in this study are followed faithfully, the person who assembles the equipment must repeat all assessment procedures of security conditions in order to avoid any undue exposure. The handling of radioactive sources requires adherence to a series of protocols that include the training of users and the use of equipment for monitoring the amount of radiation, which was not described in this article.

\section{References}

1. L. Brady, B. Micaily, C. Miyamoto, H. Heilmann, P. Montemaggi. Cancer Suppl. 76, 10 (1995)

2. N. Simon, Gynec. Oncol. 2 (1974)

3. M. Martisikova, B. Ackermann, S.Klemm, O. Jakel. Nucl. Instrum. Methods Phys. 591 (2008)

4. G. Cianguru, J. N. Yang, P.J. Oliver, M. Bues, M. Zhu, F.Nakagawa, et al. J. Appl. Clin. Med. Phys. 8 (2007)

5. H. Zhang, D. Martin, S. Chiu-Tsao, et al. Brachytherapy 9 (2010)

6. N. A. Barbosa, L.A.R. Rosa, A. Facure, D. Braz. Radiat. Phys. Chem. (to be published)

7. W.S. Santos, A.B. Carvalho, J.G. Hunt, A. F. Maia. Radiat. Phys. Chem. (to be published)

8. $\quad$ L.F. Nascimento, Y.M.M. Hornos. Radiat. Meas. 45 (2010)

9. L. Dunn, J. Lye, J. Kenny, J. Lehmann, et al. Radiat. Meas. 51 (2013)

10. J. F. Briesmeister. MCNP - A General Monte Carlo N-Particle Transport Code, Version 4C, Los Alamos National Laboratory (2000)

11. ICRP. Data Files and DEXRAX, RADSUM and WINCHAIN Codes: Corrected and Revised Codes. Based on Radionuclide Transformations: Energy and Intensity of Emissions, 38 (2003)

12. ICRP 110. Adult reference computational phantoms, International Commission on Radiological Protection (2009)

13. R.A. Cerqueira, B.M. Conceição, C.H.C. Teixeira, C.D. Mota, et al. Rev. Bras. Fís. Med. (2010)

14. D.O. Junot, D.F. Vasconcelos, D.F. Chagas, M.A.P, Santos, et al. Radiat. Meas. 46 (2011)

15. NCRP REPORT 116. Limitation of Exposure to Ionizing Radiation, National Council on Radiation Protection and Measurements (1993) 